\title{
Überprüfung von Hörsystemen Patienteninformation
}

\section{Tägliche Überprüfung von Hörsystemen - warum?}

Otoplastiken und Hörgeräte ( $\bullet$ Abb. 1 ) sind im Alltag bei Kindern besonders vielen äußeren Einflüssen ausgesetzt. Hörgeräte müssen mit in die Sandkiste, ins Planschbecken und sind beim Toben dabei. Oder sie werden aus dem Ohr gezogen, um sie mit allen Sinnen zu untersuchen und zu überprüfen. Deshalb ist es wichtig, dass Hörsysteme nicht nur beim Hörgeräteakustiker regelmäßig überprüft werden, sondern auch von allen an der Versorgung beteiligten Personen.

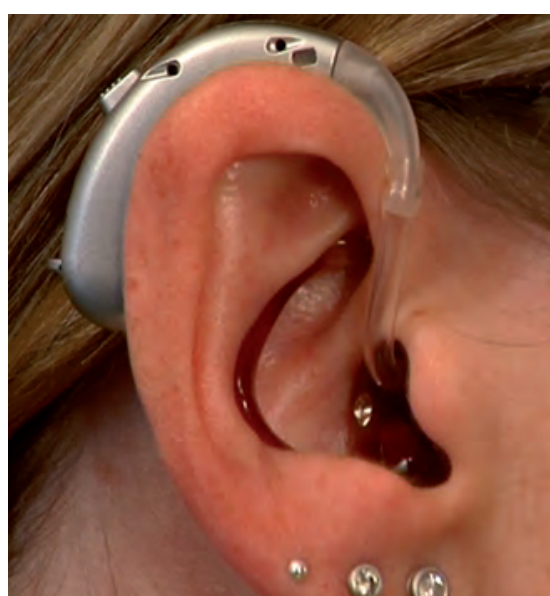

Abb. 1 Das Hörsystem besteht aus dem Hörgerät und der Otoplastik (Quelle: Akademie für Hörgeräte-Akustik, Lübeck).

In erster Linie sind es die Eltern oder weitere Bezugspersonen, die täglichen Kontakt mit dem Kind haben. Aber auch Hörgeschädigtenpädagogen, Frühförderer und Logopäden sollten vor der therapeutischen Intervention eine Überprüfung der Grundfunktion vornehmen.

\section{Tägliche Überprüfung der Hörsysteme - wie?}

Um die Otoplastik und das Hörgerät schnell zu überprüfen, empfiehlt sich eine strukturierte Vorgehensweise ( $\bullet$ Abb. 2).

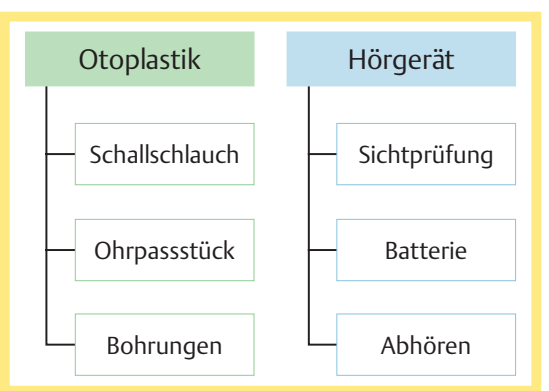

Abb. 2 Strukturiertes Protokoll für die Funktionskontrolle.

Dafür ist zuerst die Otoplastik von dem Hörgerät zu trennen. Beide sind durch einen weichen Schallschlauch miteinander verbunden. Dieser Schallschlauch verhärtet mit der Zeit, so dass zum Trennen ein fester Zug notwendig ist. Damit das Hörgerät dabei nicht beschädigt wird, hält man mit der einen Hand das Hörgerät am Hörwinkel fest, während die andere Hand dicht dahinter den Schallschlauch vom Hörwinkel abzieht (๑ Abb. 3).

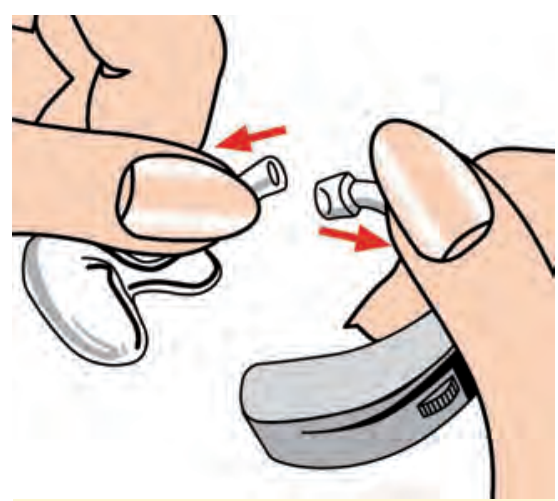

Abb. 3 Trennung des Hörgerätes von der Otoplastik (Quelle: Fa. Hansaton, Die HörsystemPflege-Fibel).

Nun hat man 2 Teile in der Hand. Die Überprüfung beginnt zuerst bei der Otoplastik.

\section{Otoplastik}

Schallschlauch Der Schallschlauch muss beweglich und frei sein. Manchmal befindet sich Feuchtigkeit im Schlauch, die mit ei- nem Trockenpuster entfernt werden muss (0 Abb.4). Weiterhin darf der Schlauch nicht durch z.B. Risse beschädigt sein.

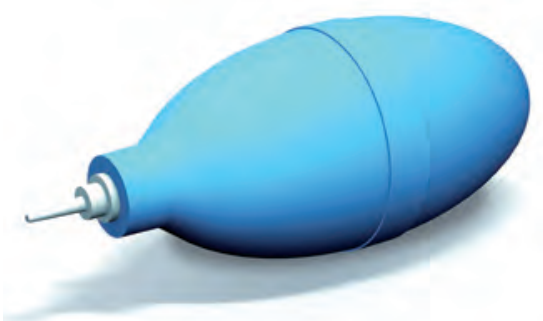

Abb. 4 Trockenpuster für die Bohrungen und den Schallschlauch (Quelle: Fa. Hansaton, Die Hörsystem-Pflege-Fibel).

Ohrpassstück Das Ohrpassstück kann in verschiedenen Formen und Farben angefertigt sein. Wichtig für die Kontrolle ist, dass keine scharfen Kanten/Ecken vorhanden sind. Manchmal können Stücke abgebrochen oder beschädigt sein, die Verletzungen im Ohr verursachen können. Nach der Kontrolle kann dann der Sitz im Ohr des Kindes überprüft werden.

Bohrungen Es können mehrere Bohrungen vorhanden sein, die alle frei und durchgängig sein müssen. Ähnlich wie im Schallschlauch können Feuchtigkeit oder hier vermehrt Cerumen die Bohrungen verstopfen und damit die Funktion erheblich beeinträchtigen.

\section{Hörgerät}

Sichtprüfung Durch eine optische Kontrolle lassen sich schon so manche Fehlerquellen finden. Sind das Gehäuse bzw. die Bedienelemente wie Umschalter und Lautstärkesteller verschmutzt oder beschädigt? Ist der Schalleintritt an der Mikrofonöffnung oder der Schallaustritt am Hörwinkel verstopft oder defekt? Vielleicht lässt sich auch die Batterielade nicht mehr schließen bzw. öffnen oder der Batteriekontakt ist verbogen oder abgebrochen. Alle diese Fehler können mit dem bloßen Auge festgestellt werden 


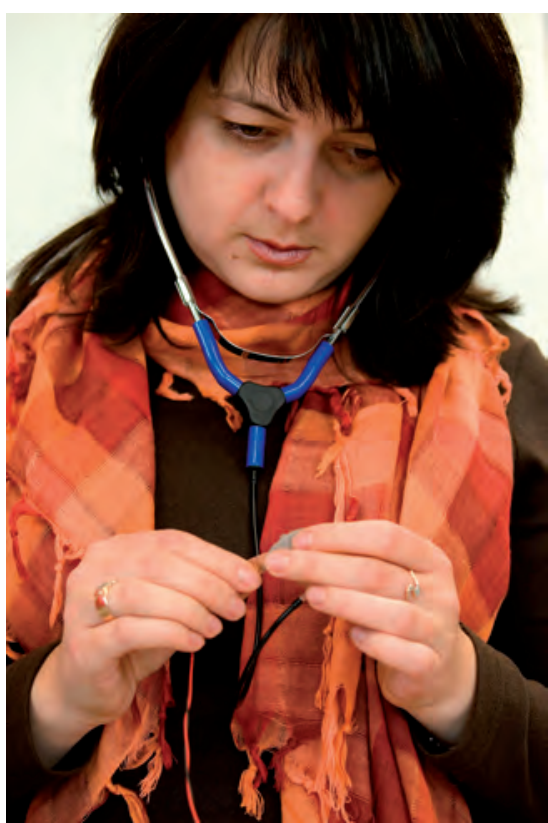

Abb. 5 Abhören mit dem Stethoclip (Quelle: Akademie für Hörgeräte-Akustik; Lübeck)

Batterie Die Batterie muss mit einem Batterietester geprüft werden. Dazu ist auf die richtige Polung zu achten (+/-). Die Spannung sollte zwischen 1,3 und $1,5 \mathrm{~V}$ liegen, bzw. bei einer optischen Anzeige im „grünen“ Bereich.

Falls kein Batterieprüfgerät zur Verfügung steht kann als Ersatzmethode ein sogenannter Rückkopplungstest durchgeführt werden. Dazu wählt man folgenden Ablauf:

1. Das Hörgerät anschalten

2. Den Lautstärkesteller (wenn aktiviert) auf ein Maximum drehen

3. Hörer und Mikrofon mit der Hand umschließen.

Sollte auf einer der 3 Stufen ein Pfeifen zu hören sein, kann man davon ausgehen, dass die Batterie noch eine Mindestspannung aufweist.

Das Rückkopplungspfeifen ist davon abhängig, bei welcher Frequenz der höchste Ausgangschalldruck vorhanden ist. Eine Aussage über die im Hörgerät vorhandene Verstärkung ist deshalb aus dieser Angabe nicht möglich. Zudem verhindern technische Regelungen, dass ein Rückkopplungspfeifen entsteht oder es wird innerhalb von Millisekunden unterdrückt. Rückkopplungsunterdrückungssysteme sind mittlerweile in fast allen digitalen Hörsystemen vorhanden.

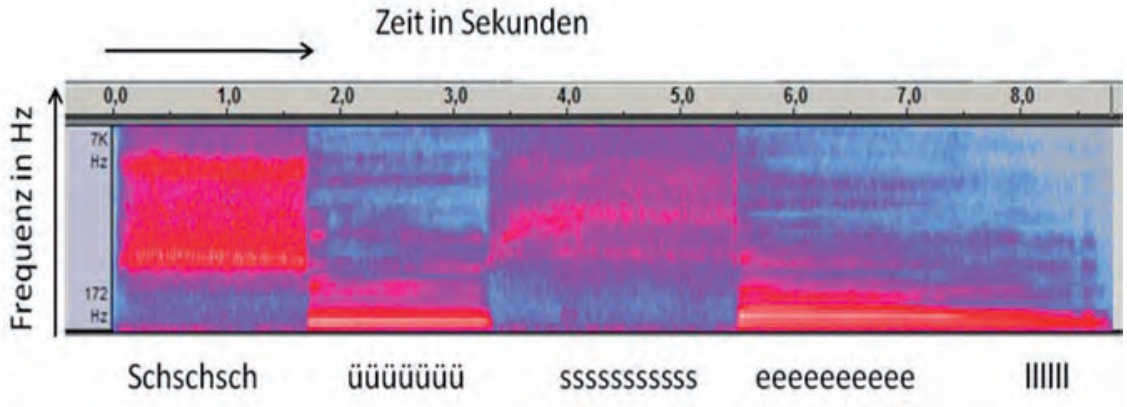

Abb. 6 Darstellung des Abhörwortes als Spektrogramm (Quelle: Akademie für Hörgeräte-Akustik, Lübeck).

Wenn Zweifel bestehen, ob die Spannungsversorgung noch ausreichend ist, sollte am besten gleich eine neue Batterie eingelegt werden.

Abhören Sollten keine sichtbaren Auffälligkeiten feststellbar sein, folgt als nächster Schritt die akustische Kontrolle. Dazu wird ein Stethoclip benötigt ( $\triangle$ Abb. 5). Die Batterie wird eingelegt und der evtl. vorhandene Lautstärkesteller auf ein Minimum gestellt, um die eigenen Ohren nicht zu schädigen. Ein eingelegter Filter/ Dämpfungselement, der in den Schlauch des Stethoclips befestigt wird, kann das Ohr zusätzlich vor zu hohen Energiespitzen schützen. Anschließend wird das Hörgerät mit dem Schallschlauch des Stethoclips verbunden.

Nach dem Einschalten wird der Lautstärkesteller langsam aufgedreht. Dieser kann bei Kindern deaktiviert sein. Nun werden einige Worte zur Überprüfung in das Mikrofon gesprochen. Hierfür eignen sich Worte wie „Schüssel“, „Schussel“ oder „Schlüssel“. Das Testwort enthält folgende akustische Anteile.

- Sch: breitbandig, hohe Energie

- U/Ü: tieftonig, hohe Energie

- SS: hochtonig, mittlere/geringe Energie

- E: mitteltonig, mittlere Energie

- L: mitteltonig, geringe Energie

(० Abb. 6)

- Sind fremde Geräusche, wie Kratzen, Brummen oder Pfeifen zu hören?

- Sind Verzerrungen wahrzunehmen?

- Setzt das Gerät bei Druck auf das Gehäuse oder bei leichtem Schütteln aus?

Sind bei dieser Prüfung keine Fehler feststellbar, kann das Hörgerät wieder mit dem Ohrpassstück verbunden und in das Ohr des Kindes eingesetzt werden. Ein kurzer Blick auf den Sitz der Otoplastik und des Hörgerätes hinter dem Ohr schließen die Kontrolle ab.

\section{Fazit}

Dieses schnelle Überprüfungsprogramm sollte von jedem, der regelmäßig mit Hörgeräteträgern zu tun hat durchgeführt werden. Damit ist eine grundlegende Basis der Kommunikation sichergestellt.

\section{Dipl.-Ing. Siegrid Meier, Lübeck}

\section{Literaturempfehlungen}

1 Akademie für Hörgeräteakustik. Berufsbegleitende Information für die Auszubildenden. Hörakustik 2009; 12: 92-94

2 Vatterschule www.vatterschule.de/content/ download/quickcheck.pdf (Zugriff am 5.10.2010)

überreicht durch Thieme und
Je höher die Energie, desto stärker ist die Rot- bzw. Weißfärbung.

In ruhiger Umgebung ist nun auf folgende akustische Auffälligkeiten zu achten:

- Wie ist die Lautstärke insgesamt?

- Lässt sich die Lautstärke regeln (bei aktiviertem Lautstärkesteller)?

- Ist die Sprache klar und deutlich zu verstehen? 